\title{
Protocol for a systematic review, meta- analysis, and trial sequential analysis of clinical outcomes following accelerated versus conventional corneal collagen cross-linking for corneal ectasia
}

\author{
Siddharth Nath ${ }^{1,2^{*}}$ (D) Carl Shen ${ }^{1}$, Alex Koziarz ${ }^{3}$ and Laura Banfield ${ }^{4}$
}

\begin{abstract}
Background: Collagen cross-linking $(\mathrm{CXL})$ is an evolving procedure that enhances the biomechanical rigidity of the cornea and can slow or halt ectatic disease. CXL requires delivery of $5.4 \mathrm{~J} / \mathrm{cm}^{2}$ of ultraviolet A (UVA) radiation to a cornea saturated with riboflavin in order to induce cross-link formation. The conventional CXL procedure achieves this fluence by exposing the cornea to a $3 \mathrm{~mW} / \mathrm{cm}^{2}$ UVA lamp for $30 \mathrm{~min}$; however, some surgeons have proposed accelerated protocols which achieve the same fluence in a shorter period of time by using a higher power light source. Whether accelerated protocols are as effective in arresting ectasia as the established conventional procedure remains unclear. Accordingly, this study will systematically review and synthesise the evidence on accelerated CXL and compare it to the conventional approach across an array of clinical outcomes.
\end{abstract}

Methods: We will search 16 electronic databases, including MEDLINE, Embase, and the Cochrane Library, from inception to February 1, 2019. We will include all randomised controlled trials comparing accelerated and conventional CXL for any corneal ectatic disease. Two reviewers will independently screen search results to identify eligible articles, complete data collection, and conduct quality assessment. The quality of individual trials will be assessed using the Cochrane Collaboration's Risk of Bias Assessment Tool. Our primary outcome will be the change in maximal keratometry $\left(K_{\max }\right)$ at 12 months following treatment. Additional outcomes will include: incidence of disease progression, incidence of serious adverse events, as well as change in $K_{\max }$ at longest follow-up, mean stromal demarcation line depth, mean uncorrected distance visual acuity, mean corrected distance visual acuity, mean $K_{\text {max }}$ mean endothelial cell density, mean central corneal thickness, mean spherical equivalent, mean intraocular pressure, and mean corneal power, at 12 months following treatment. We will calculate relative risks and 95\% confidence intervals (Cls) for dichotomous outcomes and weighted mean differences and corresponding 95\% Cls for continuous outcomes. Prespecified subgroup analyses will be performed to investigate heterogeneity. We will rate the overall quality of evidence using the Grading of Recommendations Assessment, Development, and Evaluation (GRADE) approach.

(Continued on next page)

\footnotetext{
* Correspondence: siddharth.nath@medportal.ca

${ }^{1}$ Division of Ophthalmology, Department of Surgery, McMaster University, Hamilton, Ontario, Canada

${ }^{2}$ Department of Biochemistry and Biomedical Sciences, Michael G. DeGroote

School of Medicine, Faculty of Health Sciences, McMaster University, 1200

Main Street West, Room 4N53, Hamilton, Ontario L8N 3Z5, Canada

Full list of author information is available at the end of the article
}

(c) The Author(s). 2019 Open Access This article is distributed under the terms of the Creative Commons Attribution 4.0 International License (http://creativecommons.org/licenses/by/4.0/), which permits unrestricted use, distribution, and reproduction in any medium, provided you give appropriate credit to the original author(s) and the source, provide a link to the Creative Commons license, and indicate if changes were made. The Creative Commons Public Domain Dedication waiver (http://creativecommons.org/publicdomain/zero/1.0/) applies to the data made available in this article, unless otherwise stated. 
(Continued from previous page)

Discussion: This review will provide a comprehensive evaluation of the evidence on accelerated and conventional $\mathrm{CXL}$ approaches and serve to inform clinical practice, medical device design, and future research. Evaluating variations of the $C X L$ protocol aimed at reducing treatment duration is of critical importance and a prerequisite to expanding treatment availability to more patients.

Systematic review registration: PROSPERO CRD42018104151

Keywords: Cross-linking, Accelerated, Corneal ectasia, Keratoconus, Systematic review

\section{Background}

Corneal ectasias are progressive, degenerative ocular diseases which result from thinning, bulging, and structural changes in the peripheral, central, or paracentral cornea. These structural alterations distort vision, restrict lens fitting, and result in a substantial reduction in quality of life [1]. The most common corneal ectasia is keratoconus, which affects approximately 1 in 375 individuals [2]. Despite extensive research into the cellular, molecular, and genetic mechanisms underlying ectatic processes, the pathophysiology of these diseases remains unclear, and there is a paucity of disease-modifying treatments [3]. Although many patients with ectasia may initially be managed with spectacles or rigid gas permeable lenses, a subset can have continued disease progression that requires a corneal graft [1].

Corneal collagen cross-linking (CXL) is a minimally invasive procedure proposed in the late twentieth century as a potential disease-modifying intervention for keratoconus [4]. The aim of CXL is to increase the biomechanical rigidity of the cornea by inducing cross-links both within and between collagen fibrils in the corneal stroma. This is achieved by using riboflavin as a photosensitiser, which, upon exposure to ultraviolet A (UVA) light, induces cross-link formation via the natural lysyl oxidase pathway [4, 5]. These increased cross-links serve to stabilise the structure of the cornea and enhance its stiffness, thereby protecting against further degeneration. Animal studies investigating the effect of CXL on corneal structure have been largely supportive of this hypothesis, finding significantly increased biomechanical rigidity in treated corneas [6-8]. CXL was approved for treating keratoconus and other corneal ectasias in the early 2000s across Europe and in 2016 in the USA [9, 10].

The conventional approved CXL technique, commonly referred to as the 'Dresden protocol', begins with removal of the central $7 \mathrm{~mm}$ of corneal epithelium, followed by the administration of riboflavin both prior to and during exposure of the cornea to UVA light. A fluence of $5.4 \mathrm{~J} /$ $\mathrm{cm}^{2}$ was determined to be ideal for cross-link formation, and this protocol achieves it through exposure to a 3 $\mathrm{mW} / \mathrm{cm}^{2}$ lamp for $30 \mathrm{~min}$ [9]. Multiple aspects of the conventional protocol have been modified by surgeons in efforts to refine the procedure and achieve improved safety and efficacy.

In particular, clinicians have focused on minimising the UVA light exposure time, in order to decrease the duration of the procedure. Harnessing the Bunsen-Roscoe law of reciprocity, which states that a biological effect is proportional only to the energy dose and not the method of energy delivery, some surgeons have introduced accelerated CXL [11]. This modified CXL protocol achieves the same $5.4 \mathrm{~J} / \mathrm{cm}^{2}$ fluence of UVA radiation in a shorter duration of time by implementing a stronger UVA light source. The decrease in exposure time is inversely proportional to the increase in UVA light power (e.g., $3 \mathrm{~mW} / \mathrm{cm}^{2}$ for $30 \mathrm{~min}$ translates to $9 \mathrm{~mW} / \mathrm{cm}^{2}$ for $10 \mathrm{~min}$ ). Shortening the treatment duration may improve patient comfort and minimise adverse events linked to prolonged corneal exposure [12]. Moreover, with a shorter procedure, clinicians can rapidly expand treatment availability to a greater number of patients and intervene to arrest progressive ectasia more swiftly.

\section{Why it is important to do this review}

Although accelerated CXL is rooted in principles of physics and offers considerable theoretical benefits, it remains unclear whether it induces similar biomechanical changes in the cornea as the conventional protocol. Whether the increased UVA light power is associated with additional adverse events is also uncertain. Randomised trials and observational studies comparing accelerated and conventional CXL have shown promising results; however, the field has yet to reach a consensus $[13,14]$. Thus, we will undertake a comprehensive systematic review and meta-analysis in order to evaluate the safety and efficacy of accelerated CXL in comparison to the conventional protocol.

\section{Methods \\ Objective}

This systematic review and meta-analysis will examine the safety and efficacy of accelerated CXL in comparison to conventional CXL across an array of clinically important outcomes. 


\section{Registration}

This study protocol has been developed in line with the Preferred Reporting Items for Systematic Reviews and Meta-Analysis Protocols (PRISMA-P) [15] statement and is registered with the International Prospective Register of Systematic Reviews (PROSPERO; registration number: CRD42018104151). We will conduct our systematic review and meta-analysis in accordance with the Preferred Reporting Items for Systematic Reviews and Meta-Analyses (PRISMA) [16, 17] guidelines and the Cochrane Collaboration's Handbook for Systematic Reviews of Interventions [18]. If there are insufficient randomised trials (fewer than three) available to conduct a systematic review and meta-analysis, we will include observational studies and implement guidelines set forth by the Meta-analysis of Observational Studies in Epidemiology (MOOSE) consensus statement [19]. This protocol will be updated as required by PRISMA-P criteria [15], and amended versions will be made available on PROSPERO.

\section{Literature search}

We will conduct a detailed search of the following electronic databases: MEDLINE, Embase, Web of Science,
Cochrane CENTRAL, Cochrane DSR, CINAHL, OpenGrey, metaRegister of Controlled Trials, LILACS, ClinicalTrials.gov, WHO Clinical Trials Database, WangFangData, CQVIP, INSPEC, COMPENDEX, and CNKI, from inception through February 1, 2019. We will use medical subject heading $(\mathrm{MeSH})$ terms and keywords related to corneal ectasia and the cross-linking approach (accelerated or conventional) as well as clinical outcomes. Search strategies will be designed by a multidisciplinary research team composed of clinicians, researchers, and academic librarians with expertise in conducting systematic reviews. The proposed search strategy for the MEDLINE database is provided in Table 1. Our electronic search will be supplemented by manually screening the references of eligible articles, reviewing the proceedings of relevant meetings, and contacting clinical experts in the field. We will conduct our search without any restrictions on publication type, language, or time.

\section{Study selection}

Search results will be evaluated independently by two reviewers (SN, CS) against predefined eligibility criteria to identify relevant studies (Fig. 1). Results from all searched databases will be exported as .RIS, .XML, or

Table 1 Search strategy for the MEDLINE electronic database using the Ovid interface

\begin{tabular}{|c|c|}
\hline Database & Search terms \\
\hline MEDLINE 1946-present & $\begin{array}{l}1 \text { Cross-Linking Reagents/ } \\
2 \text { CORNEA/ } \\
3 \text { cornea*.mp. } \\
41 \text { and ( } 2 \text { or } 3 \text { ) } \\
5 \text { ((crosslink* or cross link* or xlink* or x link*) adj3 (cornea* or collagen)).mp. } \\
6 \text { CCL.mp. } \\
7 \text { CXL.mp. } \\
8 \text { C3R.mp. } \\
9 \text { or/4-8 } \\
10 \text { Dilatation, Pathologic/ } \\
11 \text { ectasia*.mp. } \\
12 \text { corneal diseases/ } \\
13 \text { cornea*.mp. } \\
14 \text { exp. Corneal Dystrophies, Hereditary/ } \\
15 \text { pellucid marginal degeneration.mp. } \\
16 \text { (fuch* adj2 atroph*).mp. } \\
17 \text { ((fuch* or groenouw*) adj2 dystroph*).mp. } \\
18 \text { KERATOCONUS/ } \\
19 \text { keratoconus.mp. } \\
20 \text { Corneal Endothelial Cell Loss/ } \\
21 \text { Corneal Wavefront Aberration/ } \\
22 \text { keratopath*.mp. } \\
23 \text { or/10-22 } \\
249 \text { and } 23 \\
25 \text { "Transendothelial and Transepithelial Migration"/ } \\
26 \text { trans-epitheli*.mp. } \\
27 \text { transepitheli*.mp. } \\
28 \text { epitheli*.mp. } \\
29 \text { (dresden or phototherapeutic or photo-therapeutic or iontophoresis or tight junctions).mp. } \\
30 \text { or/25-29 } \\
3124 \text { and } 30 \\
32 \text { remove duplicates from } 31 \\
33 \text { (accelerat* or nonaccelerat* or conventional or regular or rapid or flash-linking or KXL).mp. } \\
3424 \text { and } 33 \\
35 \text { remove duplicates from } 34\end{array}$ \\
\hline
\end{tabular}




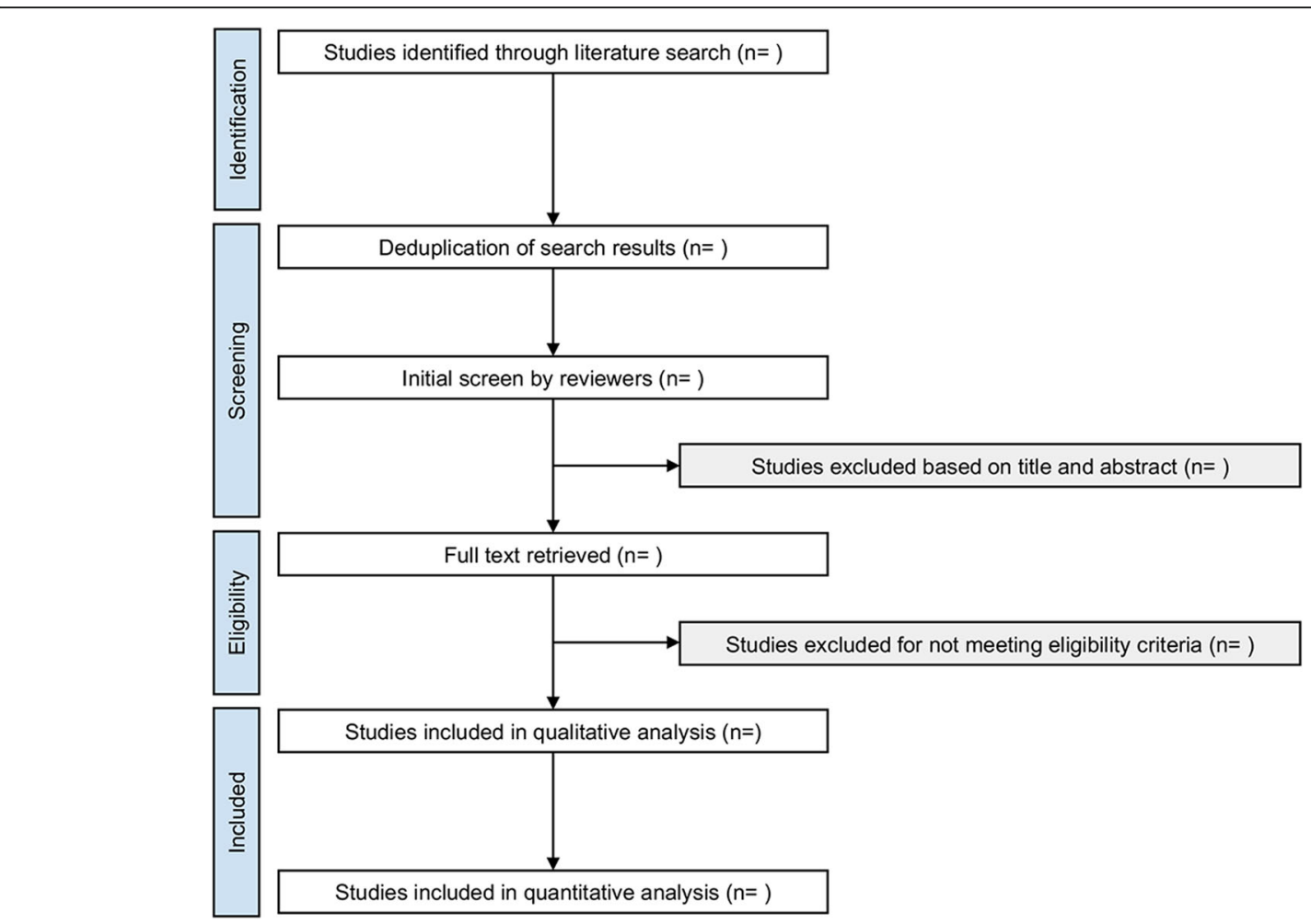

Fig. 1 PRISMA Flow Diagram

.CIW files containing the complete reference, and EndNote X9 (Clarivate Analytics, Philadelphia, USA) software will be used for reference management. Reviewers will screen titles and abstracts against inclusion criteria, and full articles will be retrieved for all references that meet these criteria, or where there is any ambiguity. In cases of ambiguity, the complete report of the specific study will be screened independently by both reviewers in order to reach a judgement on inclusion. Disagreements between reviewers will be resolved by deliberation and consensus, and, if needed, including an impartial third reviewer (AK), or contacting the trial authors.

\section{Eligibility criteria}

Our inclusion criteria will be:

- Population: patients of any demographic undergoing CXL for treatment of corneal ectasia following refractive surgery, keratoconus, or pellucid marginal degeneration.

- Intervention: accelerated CXL

- Control: conventional CXL

- Outcomes: clinical outcomes such as change in maximal keratometry $\left(K_{\max }\right)$ at 12 months after treatment (primary outcome), incidence of serious adverse events, as well as incidence of disease progression, change in $K_{\max }$ at longest follow-up, mean stromal demarcation line depth, mean $K_{\max }$, mean uncorrected distance visual acuity (UDVA), mean corrected distance visual acuity (CDVA), mean endothelial cell density, mean central corneal thickness, mean spherical equivalent, mean intraocular pressure, and mean corneal power, at 12 months following treatment.

- Study design: randomised controlled trials (including cluster trials and pilot studies) comparing accelerated and conventional CXL, with no publication type, language, or time restrictions. We will include both full articles and conference abstracts in the grey literature. Abstracts will be included only if they fulfil our eligibility criteria and if no subsequent study has been published. If we encounter duplicate studies, we will include only the report with the most current and complete data. We will exclude observational studies, unless insufficient (fewer than three) randomised trials are found, narrative reviews, systematic reviews, in vitro studies, letters to the editor and correspondences, and randomised trials examining only one CXL approach (accelerated or conventional) without a comparator. If insufficient (fewer than three) randomised controlled trials are available, we will include observational studies (prospective cohort only). Studies that examine different CXL approaches for indications other than corneal ectasia (e.g., 
infection) will also be excluded. We will record reasons for excluding studies.

\section{Data management and collection}

Data from included studies will be collected independently by two reviewers (SN, CS) and confirmed for accuracy by a third reviewer (AK). Prior to data extraction, the complete reports of all studies meeting our inclusion criteria will be collated, and reviewers will develop and pilot data extraction forms. For studies not published in English, the complete article will be translated into English and a clinical expert fluent in the original language of the study will be consulted. Discrepancies in data extraction will be resolved collaboratively by discussion amongst the two primary reviewers ( $\mathrm{SN}, \mathrm{CS}$ ), conferring with an independent third reviewer (AK), or contacting the original trial authors. Where data in included studies is incomplete or ambiguous, we will contact study authors for further information and clarification.

We will extract data from eligible studies using forms with fields for the following: study first author, year of publication, journal of publication, language, study design, included centres, included countries, number of patients, number of males, number of females, recruitment period, eligibility criteria, method of randomisation, indication for CXL, number of patients in accelerated and conventional groups, age of patients in accelerated and conventional groups, procedure for follow-up, number of patients with disease progression 12 months following CXL in accelerated and conventional groups, $K_{\max }$ before CXL in accelerated and conventional groups, $K_{\max }$ 12 months after CXL in accelerated and conventional groups, $K_{\max }$ at longest follow-up after CXL in accelerated and conventional groups, UDVA before CXL in accelerated and conventional groups, UDVA 12 months after CXL in accelerated and conventional groups, CDVA before CXL in accelerated and conventional groups, CDVA 12 months after CXL in accelerated and conventional groups, central corneal thickness before CXL in accelerated and conventional groups, central corneal thickness 12 months after CXL in accelerated and conventional groups, endothelial cell density before CXL in accelerated and conventional groups, endothelial cell density 12 months after CXL in accelerated and conventional groups, intraocular pressure before CXL in accelerated and conventional groups, intraocular pressure 12 months after CXL in accelerated and conventional groups, corneal power before CXL in accelerated and conventional groups, corneal power 12 months after CXL in accelerated and conventional groups, spherical equivalent before CXL in accelerated and conventional groups, spherical equivalent 12 months after CXL in accelerated and conventional groups, stromal demarcation line depth after CXL in accelerated and conventional groups, method for epithelium removal, method for transepithelial riboflavin application, postoperative pain following accelerated and conventional CXL, time to best CDVA following accelerated and conventional CXL, time to best UDVA following accelerated and conventional CXL, power of UVA light in accelerated group, UVA light exposure time in accelerated group, incidence of significant complications (e.g., corneal melt, persistent epithelial defects, scarring, and persistent stromal haze) after accelerated and conventional CXL, and protocol preference as reported by authors.

\section{Risk of bias in individual studies}

All included trials will be assessed using the Cochrane Collaboration's Risk of Bias Assessment Tool by two independent authors (SN, CS) [20]. Studies will be assessed to determine the risk of selection, performance, detection, attrition, reporting, and other biases. Discrepancies in quality assessment will be resolved collegially by deliberation and consensus, and consultation with an impartial third reviewer (AK). If there is insufficient information available to make a judgement on risk within an individual domain, we will rank that domain as 'unclear' and the original trial authors will be contacted for further information. Studies with one or more domains assessed to be 'high risk' will be categorised as having an overall high risk of bias.

\section{Definition of outcomes}

Our primary outcome will be the change in $K_{\max }$ (in dioptres, D) over 12 months. Additional outcomes shall be the following: incidence of disease progression (defined as an increase of the $K_{\max }$ by $\geq 1.0$ dioptres (D) over 12 months) after treatment, as well as change in: mean UDVA (in $\log$ MAR) at 12 months following treatment, mean CDVA (in logMAR) at 12 months following treatment, mean endothelial cell density (in cells/ $\mathrm{mm}^{2}$ ) at 12 months following treatment, mean $K_{\max }$ (D) at longest follow-up after treatment, mean stromal demarcation line depth (in $\mu \mathrm{m}$ ) after treatment, mean central corneal thickness (in $\mu \mathrm{m}$ ) at 12 months following treatment, mean spherical equivalent (D) at 12 months following treatment, mean intraocular pressure (in $\mathrm{mmHg}$ ) at 12 months following treatment, mean corneal power (D) at 12 months following treatment, and the mean $K_{\max }(\mathrm{D})$ at 12 months after treatment. We will also examine the incidence of serious adverse events (e.g., corneal melt, persistent epithelial defects, scarring, and persistent stromal haze).

\section{Data synthesis}

Analyses will be conducted by the intention-to-treat principle using pooled study-level data. For dichotomous outcomes, such as incidence of ectatic disease 
progression and incidence of serious adverse events, we will summarise our analyses by calculating the relative risk (RR) with corresponding 95\% confidence interval (CI). For continuous outcomes, such as change in $K_{\max }$, mean $K_{\text {max }}$, mean UDVA, mean CDVA, mean central corneal thickness, mean stromal demarcation line depth, mean endothelial cell density, mean intraocular pressure, mean corneal power, and mean spherical equivalent, at 12 months following treatment, we will compute the weighted mean difference (MD) with 95\% CI. Moreover, we will determine pooled estimates of all incidences across studies for the accelerated group, and then separately for the conventional group. The DerSimonian and Laird random effects model will be used to conduct our meta-analysis, and weights will be calculated using the inverse variance method [21]. The threshold for type I error for statistical significance shall be $\alpha=0.05$. Between-study heterogeneity will be assessed using Cochran's $Q$ test and quantified by the $I^{2}$ statistic, with $I^{2}$ values in excess of $25 \%, 50 \%$, and $75 \%$, graded as low, moderate, and high heterogeneity, respectively [22]. Publication bias will be examined qualitatively by visual inspection of funnel plot symmetry and quantified by Begg and Mazumdar's [23] and Egger's tests [24].

We will undertake prespecified subgroup analyses to investigate whether covariates exist and to examine heterogeneity in our primary outcome. Analyses will be performed for subgroups stratified by patient age and sex, CXL technique (transepithelial versus epithelium-off), severity of disease at time of treatment, and study risk of bias (low versus high). In addition, we will conduct a sensitivity analysis by performing trial sequential analysis (TSA) $[25,26]$. TSA adjusts for the risk of type I error and constructs monitoring boundaries to determine whether an intervention is beneficial, harmful, or futile in comparison to control. We will perform TSA with an overall power of $80 \%$ and type I error risk of $5 \%$.

The quality of available evidence will be summarised using the Grading of Recommendations Assessment, Development, and Evaluation (GRADE) approach [27]. Evidence will be assessed for the domains of risk of bias, consistency, precision, reporting bias, directness, and other bias. The evidence for each outcome will be ranked as being of high, moderate, low, or very low quality in accordance with criteria set by the GRADE working group.

All statistical analyses will be conducted using Comprehensive Meta-analysis v.3.3.070 (Biostat, Englewood, New Jersey, USA), and TSA will be performed using Trial Sequential Analysis v0.9.5.10 Beta (Copenhagen Trial Unit, Copenhagen, Denmark).

\section{Discussion}

The results of this review are expected to considerably inform clinical practice, future research, and the management of patients with ectatic disease. By comprehensively examining the evidence on accelerated and conventional CXL approaches, this study is expected to influence how clinicians perform this evolving treatment and how UVA cross-linking devices are engineered and, potentially, improve patient outcomes. Moreover, through analysing the safety and efficacy of accelerated CXL, this study may incite changes leading to shorter operative times and increased treatment availability. Findings from this study will be submitted to a peer-reviewed journal for publication and will be presented at conferences and seminars.

\section{Abbreviations \\ CDVA: Corrected distance visual acuity; Cl: Confidence interval; CXL: Corneal collagen cross-linking; GRADE: Grading of Recommendations Assessment, Development, and Evaluation; $K_{\max }$ : Maximal keratometry; MD: Mean difference; MeSH: Medical subject heading; PRISMA: Preferred Reporting Items for Systematic Review and Meta-Analyses; PRISMA-P: Preferred Reporting Items for Systematic Review and Meta-Analysis Protocols; PROSPERO: International Prospective Register of Systematic Reviews; RR: Relative risk; TSA: Trial sequential analysis; UDVA: Uncorrected distance visual acuity; UVA: Ultraviolet A}

\section{Acknowledgements}

Not applicable.

\section{Funding}

This research received no specific grant from any funding agency in the public, private, or not-for-profit sectors.

\section{Availability of data and materials}

Data sharing is not applicable to this article as no datasets were generated or analysed during the current study.

\section{Authors' contributions \\ SN and CS conceived and designed the study. SN and LB developed the search strategy and SN, LB, and CS piloted it in electronic databases. SN and CS developed the review protocol, eligibility criteria, risk of bias assessment strategy, and data management processes. SN, CS, and AK established the data synthesis methodology. All authors critically revised and commented on the intellectual content of the manuscript. All authors read and approved the final manuscript.}

Ethics approval and consent to participate

Not applicable.

Consent for publication

Not applicable.

\section{Competing interests}

The authors declare that they have no competing interests.

\section{Publisher's Note}

Springer Nature remains neutral with regard to jurisdictional claims in published maps and institutional affiliations.

\section{Author details}

${ }^{1}$ Division of Ophthalmology, Department of Surgery, McMaster University, Hamilton, Ontario, Canada. ${ }^{2}$ Department of Biochemistry and Biomedical Sciences, Michael G. DeGroote School of Medicine, Faculty of Health Sciences, McMaster University, 1200 Main Street West, Room 4N53, Hamilton, Ontario L8N 3Z5, Canada. ${ }^{3}$ Faculty of Medicine, University of Toronto, Toronto, Ontario, Canada. ${ }^{4}$ Health Sciences Library, McMaster University, Hamilton, Ontario, Canada. 
Received: 26 September 2018 Accepted: 25 March 2019

Published online: 04 April 2019

\section{References}

1. Maharana PK, Dubey A, Jhanji V, Sharma N, Das S, Vajpayee RB. Management of advanced corneal ectasias. Br J Ophthalmol. 2016;100:34-40.

2. Godefrooij DA, de Wit GA, Uiterwaal CS, Imhof SM, Wisse RP. Age-specific incidence and prevalence of keratoconus: a nationwide registration study. Am J Ophthalmol. 2017;175:169-72.

3. Romero-Jiménez M, Santodomingo-Rubido J, Wolffsohn JS. Keratoconus: a review. Cont Lens Anterior Eye. 2010;33:157-66.

4. Spoerl E, Huhle M, Seiler T. Induction of cross-links in corneal tissue. Exp Eye Res. 1998;66:97-103.

5. O'Brart DPS. Corneal collagen cross-linking: a review. J Optom. 2014;7:113-24.

6. Spoerl E, Huhle M, Seiler T. Erhöhung der Festigkeit der Hornhaut durch Vernetzung. Ophthalmologe. 1997;94:902-6.

7. Bueno JM, Avila FJ, Martinez-Garcia MC. Quantitative Analysis of the Corneal Collagen Distribution after In Vivo Cross-Linking with Second Harmonic Microscopy. BioMed Research international. 2019;1-12. https://doi.org/10. 1155/2019/3860498.

8. Spoerl E, Schreiber J, Hellmund K, Seiler T, Knuschke P. Untersuchungen zur Verfestigung der Hornhaut am Kanichen. Ophthalmologe. 2000;97:203-6.

9. Wollensak G, Spoerl E, Seiler T. Riboflavin/ultraviolet-a-induced collagen crosslinking for the treatment of keratoconus. Am J Ophthalmol. 2003;135:620-7.

10. Mohammadpour M, Masoumi A, Mirghorbani M, Shahraki K, Hashemi H. Updates on corneal collagen cross-linking: indications, techniques and clinical outcomes. J Curr Ophthalmol. 2017;29:235-47.

11. Bunsen RW, Roscoe BA. Photochemical researches - part V. On the measurement of the chemical action of direct and diffuse sunlight. Proc $R$ Soc Lond. 1862;12:306-12.

12. Woo JH, lyer JV, Lim L, et al. Conventional versus accelerated collagen crosslinking for keratoconus: a comparison of visual, refractive, topographic and biomechanical outcomes. Open Ophthalmol J. 2017:11:262-72.

13. Sadoughi MM, Einollahi B, Baradaran-Rafii A, Roshandel D, Hasani H, Nazeri M. Accelerated versus conventional corneal collagen cross-linking in patients with keratoconus: an intrapatient comparative study. Int Ophthalmol. 2018;38:67-74

14. Elbaz U, Shen C, Lichtinger A, et al. Accelerated $\left(9-\mathrm{mW} / \mathrm{cm}^{2}\right)$ corneal collagen crosslinking for keratoconus-a 1-year follow-up. Cornea. 2015;33:769-73.

15. Shamseer L, Moher D, Clarke M, et al. Preferred reporting items for systematic review and meta-analysis protocols (PRISMA-P) 2015: elaboration and explanation. BMJ. 2015;350:g7647.

16. Moher D, Liberati A, Tetzlaff J, et al. Preferred reporting items for systematic reviews and meta-analyses: the PRISMA statement. BMJ. 2009;339:b2535.

17. Liberati A, Altman DG, Tetzlaff J, et al. The PRISMA statement for reporting systematic reviews and meta-analyses of studies that evaluate healthcare interventions: explanation and elaboration. BMJ. 2009;229:b2700.

18. Cochrane handbook for systematic reviews of interventions. 5.1.0 ed. The Cochrane Collaboration, 2011.

19. Stroup DF, Berlin JA, Morton SC, et al. Meta-analysis of observational studies in epidemiology: a proposal for reporting. Meta-analysis of Observational Studies in Epidemiology (MOOSE) group. JAMA. 2000;283:2008-12.

20. Higgins JP, Altman DG, Gøtzsche PC, et al. The Cochrane Collaboration's tool for assessing risk of bias in randomised trials. BMJ. 2011;343:d5928.

21. DerSimonian R, Laird N. Meta-analysis in clinical trials. Control Clin Trials. 1986;7:177-88.

22. Higgins JP, Thompson SG, Deeks JJ, et al. Measuring inconsistency in metaanalyses. BMJ. 2003;327:557-60.

23. Begg CB, Mazumdar M. Operating characteristics of a rank correlation test for publication bias. Biometrics. 1994;50:1088-101.

24. Egger M, Davey Smith G, Schneider M, et al. Bias in meta-analysis detected by a simple, graphical test. BMJ. 1997;315:629-34.

25. Brok J, Thorlund K, Gluud C, Wetterslev J. Trial sequential analysis reveals insufficient information size and potentially false positive results in many meta-analyses. J Clin Epidemiol. 2008;61:763-9.

26. Wetterslev J, Thorlund K, Brok J, Gluud C. Trial sequential analysis may establish when firm evidence is reached in cumulative meta-analysis. I Clin Epidemiol. 2008;61:64-75.

27. Guyatt $G H$, Oxman $A D$, Vist GE, et al. GRADE: an emerging consensus on rating quality of evidence and strength of recommendations. BMJ. 2008:336: 924-6.

\section{Ready to submit your research? Choose BMC and benefit from:}

- fast, convenient online submission

- thorough peer review by experienced researchers in your field

- rapid publication on acceptance

- support for research data, including large and complex data types

- gold Open Access which fosters wider collaboration and increased citations

- maximum visibility for your research: over $100 \mathrm{M}$ website views per year

At $\mathrm{BMC}$, research is always in progress.

Learn more biomedcentral.com/submissions 